\title{
Finite difference method for Riesz space fractional diffusion equations with delay and a nonlinear source term
}

\section{Shuiping Yang}

School of Mathematics and Big Data Science, Huizhou University, Guangdong, 516007, China.

Communicated by D. Baleanu

\begin{abstract}
In this paper, we propose a finite difference method for the Riesz space fractional diffusion equations with delay and a nonlinear source term on a finite domain. The proposed method combines a time scheme based on the predictor-corrector method and the Crank-Nicolson scheme for the spatial discretization. The corresponding theoretical results including stability and convergence are provided. Some numerical examples are presented to validate the proposed method. (C)2017 All rights reserved.
\end{abstract}

Keywords: Riesz fractional derivative, fractional diffusion equations, Crank-Nicolson scheme, stability, convergence.

2010 MSC: 34K28, 65M12, 35R11, 34K37.

(C)2018 All rights reserved.

\section{Introduction}

In this paper, we consider the following Riesz space fractional diffusion equations with delay and a nonlinear source term

$$
\left\{\begin{array}{l}
\frac{\partial u(x, t)}{\partial t}=K_{\alpha}(x, t) \frac{\partial^{\alpha} u(x, t)}{\partial|\alpha|^{\alpha}}+f(x, t, u, u(x, t-s)), 1<\alpha \leqslant 2,(x, t) \in(0, L) \times[0, T] \\
u(0, t)=u(L, t)=0, t \in[0, T] \\
u(x, t)=\varphi(x, t),(x, t) \in(0, L) \times[-s, 0]
\end{array}\right.
$$

where $1<\alpha \leqslant 2, s>0$, and $f: D=[0, L] \times[0, T] \times \mathbb{R} \times \mathbb{R} \rightarrow \mathbb{R}$ is a given continuous mapping and satisfies the following Lipschitz condition

$$
\left|f\left(x, t, u_{1}, u_{2}\right)-f\left(x, t, \bar{u}_{1}, \bar{u}_{2}\right)\right| \leqslant \beta_{1}\left|u_{1}-\bar{u}_{1}\right|+\beta_{2}\left|u_{2}-\bar{u}_{2}\right|, \quad \forall u_{1}, \bar{u}_{1}, u_{2}, \bar{u}_{2} \in \mathbb{R},
$$

where $\beta_{1}>0, \beta_{2}>0$, and $K_{\alpha} \geqslant 0$ represents the dispersion coefficient. The Riesz space fractional

Email address: yang52053052@163.com (Shuiping Yang)

doi: $10.22436 /$ jnsa.011.01.03

Received: 2017-08-02 Revised: 2017-11-03 Accepted: 2017-12-01 
operators $\frac{\partial^{\alpha} \mathfrak{u}(x, \mathrm{t})}{\partial|\alpha|^{\alpha}}$ on a finite domain $[0, \mathrm{~L}]$ can be defined as

$$
\frac{\partial^{\alpha} u(x, t)}{\partial|\alpha|^{\alpha}}=-c_{\alpha}\left[{ }_{0} D_{\chi}^{a} u(x, t)+{ }_{x} D_{L}^{\alpha} u(x, t)\right]
$$

where

$$
c_{\alpha}=\frac{1}{2 \cos \left(\frac{\pi \alpha}{2}\right)}
$$

and

$$
{ }_{0} D_{x}^{\alpha} u(x, t)=\frac{1}{\Gamma(2-\alpha)} \frac{\partial^{2}}{\partial x^{2}} \int_{0}^{x}(x-\tau)^{1-\alpha} u(\tau, t) d \tau, \quad{ }_{x} D_{L}^{\alpha} u(x, t)=\frac{1}{\Gamma(2-\alpha)} \frac{\partial^{2}}{\partial x^{2}} \int_{x}^{L}(\tau-x)^{1-\alpha} u(\tau, t) d \tau,
$$

where $\Gamma(\cdot)$ represents the Euler gamma function.

In the past decades, fractional calculus has been a hot spot of researches in science and engineering $[1,6,8,9,15,18,20-22]$. The numerical approach to fractional differential equations (FDEs) has been discussed by numerous researchers [2-5, 7, 10-14, 17, 19, 26]. Recently, in [23-25], some new finite memory fractional differences have been proposed. And these definitions can be considered for discrete fractional modeling. Consideration numerical methods for solving the fractional advection-dispersion equations (FADE) have been proposed. In 2004, Meerschaert and Tadjeran [16] developed practical numerical methods to solve the one-dimensional space FADE with variable coefficients on a finite domain. Liu et al. [10] proposed a class of method of lines for solving the space fractional Fokker-Planck equation in 2004. In 2009, Zhuang et al. [27] discussed a variable order fractional advection diffusion equation with a nonlinear source term on a finite domain. In addition, some numerical methods including finite difference method, finite element method, spectral method are also employed to solve the FADE [12]. Recently, these numerical methods for Riesz space fractional advection-dispersion equations (RFDEs) have also been a subject of many investigations [12].

As we all know, functional differential equations including delay differential equations have been widely used in physics, biology, medicine, chemistry, economics, and so on. In my opinion, it is valuable to study numerical methods for Riesz space fractional diffusion equations with delay. In this paper, our aim is to construct the numerical method for the Riesz space fractional diffusion equations with delay and obtain the corresponding theoretical results.

This paper is organized as follows. In Section 2, we introduce some definitions and lemmas. In Section 3 , we present the finite difference method for the Riesz space fractional diffusion equations with delay. The proofs of the stability and convergence of the method are provided in Section 4. Finally we carry out some numerical tests to verify the theoretical results in Section 5.

\section{Preliminaries}

In this section, we first review the basic definitions and some useful lemmas.

Definition 2.1 ([17]). The left and right Riemann-Liouville fractional derivatives of order $\gamma(n-1<\gamma<n)$ of the function $v(x)$ on $[a, b]$, are given by

$$
{ }_{a}^{\mathrm{R}} \mathrm{D}_{x}^{\gamma} \nu(x)=\frac{1}{\Gamma(n-\gamma)} \frac{d^{n}}{d x^{n}} \int_{a}^{x}(t-\tau)^{n-\gamma-1} v(\tau) d \tau,
$$

and

$$
{ }_{x}^{R} D_{b}^{\gamma} v(x)=\frac{1}{\Gamma(n-\gamma)} \frac{d^{n}}{d x^{n}} \int_{x}^{b}(t-\tau)^{n-\gamma-1} v(\tau) d \tau,
$$

respectively. 
In the interval $[0, L]$, we take the mesh points $x_{i}=i h, i=1,2, \ldots, M$ and $t_{n}=n \tau, n=1,2, \ldots, N$, where $h=\frac{L}{M}, \tau=\frac{T}{N}$, i.e., $h$ and $\tau$ are the uniform spatial step size and temporal step size, $s=m \tau$. Now, we give the following lemmas.

Lemma 2.2 ([12]). Supposing $1<\gamma<2$, let $v(x) \in \mathrm{L}^{1}(\mathbb{R})$, then

$$
{ }_{0}^{\mathrm{R}} \mathrm{D}_{\chi}^{\gamma} v\left(x_{i}\right)=\frac{1}{\mathrm{~h}^{\gamma}} \sum_{\mathrm{k}=0}^{\mathrm{i}+1} \omega_{k}^{\gamma} v\left(x_{i-k+1}\right)+\mathrm{O}\left(\mathrm{h}^{2}\right), \quad{ }_{x}^{\mathrm{R}} \mathrm{D}_{\chi} \mathrm{L}^{\gamma} v\left(x_{i}\right)=\frac{1}{\mathrm{~h}^{\gamma}} \sum_{\mathrm{k}=0}^{\mathrm{m}-\mathrm{i}+1} \omega_{k}^{\gamma} v\left(x_{i+k-1}\right)+\mathrm{O}\left(\mathrm{h}^{2}\right),
$$

where

$$
\omega_{0}^{(\gamma)}=\frac{\gamma}{2} g_{0}^{(\gamma)}, \quad \omega_{k}^{(\gamma)}=\frac{\gamma}{2} g_{k}^{(\gamma)}+\frac{2-\gamma}{2} g_{k-1}^{(\gamma)}, \quad g_{0}^{(\gamma)}=1, \quad g_{k}^{(\gamma)}=\left(1-\frac{\gamma+1}{k}\right) g_{k-1}^{(\gamma)}, k=1,2, \ldots
$$

In fact the coefficients $g_{k}^{(\gamma)}$ are the coefficients of the power series of the function $(1-z)^{\gamma}$ for all $|z| \leqslant 1$.

Lemma 2.3 ([12]). Supposing that $1<\gamma \leqslant 2$, then the coefficient $\mathrm{g}_{\mathrm{k}}^{(\gamma)}$ satisfies

$$
\left\{\begin{array}{l}
g_{0}^{(\gamma)}=1, \quad g_{1}^{(\gamma)}=-\gamma, \quad g_{2}^{(\gamma)}=\frac{\gamma(\gamma-1)}{2}>0, \\
1 \geqslant g_{2}^{(\gamma)} \geqslant g_{3}^{(\gamma)} \geqslant \cdots \geqslant 0, \quad \sum_{k=0}^{\infty} g_{k}^{(\gamma)}=0, \quad \sum_{k=0}^{m} g_{k}^{(\gamma)}<0, \quad m \geqslant 1
\end{array}\right.
$$

Lemma 2.4 ([12]). Supposing that $1<\gamma \leqslant 2$, then the coefficient $\omega_{\mathrm{k}}^{(\gamma)}$ satisfies

$$
\left\{\begin{array}{l}
\omega_{0}^{(\gamma)}=\frac{\gamma}{2}, \quad \omega_{1}^{(\gamma)}=\frac{2-\gamma-\gamma^{2}}{2}<0, \quad g_{2}^{(\gamma)}=\frac{\gamma\left(\gamma^{2}+\gamma-4\right)}{4} \\
1 \geqslant \omega_{2}^{(\gamma)} \geqslant \omega_{3}^{(\gamma)} \geqslant \cdots \geqslant 0, \quad \sum_{k=0}^{\infty} \omega_{k}^{(\gamma)}=0, \quad \sum_{k=0}^{m} \omega_{k}^{(\gamma)}<0, \quad m \geqslant 2 .
\end{array}\right.
$$

\section{The finite difference method for Riesz space fractional diffusion equations with delay and a non- linear source term}

In this section, we discuss the numerical method of (1.1). First, we define $t_{n}=n \tau, n=0,1, \cdots, N$, let $\Omega=[0, \mathrm{~L}]$ be a finite domain, setting $S_{h}$ be a uniform partition of $\Omega$, which is given by $x_{i}=i$ ih for $i=0,1, \cdots, M, s=m \tau$, where $\tau=\frac{T}{N}$ and $h=\frac{L}{M}$ are the time and space stepsize, respectively.

Now, we present the semi-discrete form of (1.1),

$$
\frac{\partial u\left(x_{i}, t\right)}{\partial t}=\left.K_{\alpha} \frac{\partial^{\alpha} u(x, t)}{\partial|x|^{\alpha}}\right|_{x=x_{i}}+f\left(x_{i}, t, u\left(x_{i}, t\right), u\left(x_{i}, t-s\right)\right) .
$$

Applying the predictor-corrector method and the Crank-Nicolson scheme to solve (3.1), we have

$$
\left\{\begin{aligned}
\bar{u}_{i}^{n}= & u_{i}^{n-1}-\frac{\tau K_{\alpha} c_{\alpha}}{h^{\alpha}}\left[\sum_{k=0}^{i+1} \omega_{k}^{(\alpha)} u_{i-k+1}^{n-1}+\sum_{k=0}^{M-i+1} \omega_{k}^{(\alpha)} u_{i+k-1}^{n-1}\right]+\tau f\left(x_{i}, t_{n}, \bar{u}_{i}^{n-1}, \bar{u}_{i}^{n-m}\right), \\
u_{i}^{n}= & u_{i}^{n-1}-\frac{\tau K_{\alpha} c_{\alpha}}{2 h^{\alpha}}\left[\left(\sum_{k=0}^{i+1} \omega_{k}^{(\alpha)} u_{i-k+1}^{n-1}+\sum_{k=0}^{M-i+1} \omega_{k}^{(\alpha)} u_{i+k-1}^{n-1}\right)+\left(\sum_{k=0}^{i+1} \omega_{k}^{(\alpha)} \bar{u}_{i-k+1}^{n-1}\right.\right. \\
& \left.\left.+\sum_{k=0}^{M-i+1} \omega_{k}^{(\alpha)} \bar{u}_{i+k-1}^{n-1}\right)\right]+\frac{\tau}{2}\left[f\left(x_{i}, t_{n}, u_{i}^{n-1}, u_{i}^{n-m}\right)+f\left(x_{i}, t_{n}, \bar{u}_{i}^{n-1}, u_{i}^{n-m}\right)\right],
\end{aligned}\right.
$$


where $u_{i}^{n}$ is approximate solution of $u\left(x_{i}, t_{n}\right)$. Denote

$$
A=\left[\begin{array}{cccccc}
\omega_{1}^{(\alpha)} & \omega_{0}^{(\alpha)} & 0 & \cdots & 0 & 0 \\
\omega_{2}^{(\alpha)} & \omega_{1}^{(\alpha)} & \omega_{0}^{(\alpha)} & \cdots & 0 & 0 \\
\omega_{3}^{(\alpha)} & \omega_{2}^{(\alpha)} & \omega_{1}^{(\alpha)} & \cdots & 0 & 0 \\
\vdots & \vdots & \vdots & \ddots & \vdots & \vdots \\
\omega_{M-2}^{(\alpha)} & \omega_{M-3}^{(\alpha)} & \omega_{M-4}^{(\alpha)} & \cdots & \omega_{1}^{(\alpha)} & \omega_{0}^{(\alpha)} \\
\omega_{M-1}^{(\alpha)} & \omega_{M-2}^{(\alpha)} & \omega_{M-3}^{(\alpha)} & \cdots & \omega_{2}^{(\alpha)} & \omega_{1}^{(\alpha)}
\end{array}\right], \quad F(U)=\left[\begin{array}{c}
f\left(x_{1}, t_{n}, u_{1}, u_{1}^{n-m}\right) \\
f\left(x_{2}, t_{n}, u_{2}, u_{2}^{n-m}\right) \\
\vdots \\
f\left(x_{M-1}, t_{n}, u_{M-1}, u_{M-1}^{n-m}\right]
\end{array}\right]
$$

and $D=-\frac{\tau K_{\alpha} c_{\alpha}}{h^{\alpha}}\left(A+A^{\top}\right), u^{n}=\left[u_{1}^{n}, u_{2}^{n}, \cdots, u_{M-1}^{n}\right], \bar{u}^{n}=\left[\bar{u}_{1}^{n}, \bar{u}_{2}^{n}, \cdots, \bar{u}_{M-1}^{n}\right]$. Thus, (3.2) can be simplified as

$$
\left\{\begin{array}{l}
\bar{u}^{n}=U^{n-1}+D U^{n-1}+\tau F\left(U^{n-1}\right), \\
u^{n}=U^{n-1}+\frac{1}{2} D\left(U^{n-1}+\bar{U}^{n}\right)+\frac{\tau}{2}\left(F\left(U^{n-1}\right)+F\left(\bar{U}^{n}\right)\right) .
\end{array}\right.
$$

Obviously, the above scheme can be written as the following scheme

$$
\mathrm{U}^{\mathrm{n}}=\mathrm{U}^{\mathrm{n}-1}+\frac{1}{2} \mathrm{D}\left(2 \mathrm{U}^{\mathrm{n}-1}+\mathrm{D} \mathrm{U}^{\mathrm{n}-1}+\tau \mathrm{F}\left(\mathrm{U}^{\mathrm{n}-1}\right)\right)+\frac{\tau}{2}\left(\mathrm{~F}\left(\mathrm{U}^{\mathrm{n}-1}\right)+\mathrm{F}\left(\mathrm{U}^{\mathrm{n}-1}+\mathrm{DU}^{\mathrm{n}-1}+\tau \mathrm{F}\left(\mathrm{U}^{\mathrm{n}-1}\right)\right)\right) .
$$

The boundary and initial conditions are discretized as

$$
u_{i}^{0}=\varphi(i h, 0), \quad u^{0}=\left(u_{1}^{0}, u_{2}^{0}, \ldots, u_{M-1}^{0}\right), i=1,2, \ldots, M-1, \quad \text { and } \quad u_{i}^{k}=\varphi(i h, k \tau), k<0 .
$$

\section{Theoretical analysis of the finite difference method}

In this section, we present the proofs of stability and convergence of the proposed method.

Lemma 4.1 ([12]). The matrix $\mathrm{D}$ is symmetric positive definite.

Theorem 4.2 (Stability). If $\|\mathrm{I}+\mathrm{D}\|_{\infty} \leqslant 1$, the method (3.3) is stable.

Proof. Let $u_{i}^{n}$ and $\tilde{u}_{i}^{n}$ be the numerical and approximate solution vectors, respectively, and

$$
\tilde{u}^{n}=\left[\tilde{u}\left(x_{1}, t_{n}\right), \tilde{u}\left(x_{2}, t_{n}\right), \cdots, \tilde{u}\left(x_{M-1}, t_{n}\right)\right]^{\top} \text {, and } \epsilon^{n}=u^{n}-u^{n} .
$$

Then,

$$
\begin{aligned}
\epsilon^{n}= & \epsilon^{n-1}+\frac{1}{2} D\left(2 \epsilon^{n-1}+D \epsilon^{n-1}+\tau\left(F\left(U^{n-1}\right)-F\left(u^{n-1}\right)\right)\right) \\
& +\frac{\tau}{2}\left[\left(F\left(U^{n-1}\right)+F\left(U^{n-1}+D u^{n-1}+\tau F\left(U^{n-1}\right)\right)\right)-\left(F\left(u^{n-1}\right)+F\left(u^{n-1}+D u^{n-1}+\tau F\left(u^{n-1}\right)\right)\right)\right] \\
= & \left(I+D+\frac{D^{2}}{2}\right) \epsilon^{n-1}+\frac{\tau}{2} D\left(F\left(U^{n-1}\right)-F\left(u^{n-1}\right)\right) \\
& +\frac{\tau}{2}\left[F\left(U^{n-1}\right)-F\left(u^{n-1}\right)+F\left((I+D) u^{n-1}+\tau F\left(U^{n-1}\right)\right)-F\left((I+D) u^{n-1}+\tau F\left(u^{n-1}\right)\right)\right] .
\end{aligned}
$$

Since $\|\mathrm{I}+\mathrm{D}\|_{\infty} \leqslant 1$, then

$$
\left\|\mathrm{I}+\mathrm{D}+\frac{1}{2} \mathrm{D}^{2}\right\|_{\infty}=\left\|\frac{1}{2}\left(\mathrm{I}+(\mathrm{I}+\mathrm{D})^{2}\right)\right\|_{\infty} \leqslant \frac{1}{2}\left(1+\|\mathrm{I}+\mathrm{D}\|_{\infty}^{2}\right) \leqslant 1 .
$$

By using the condition (1.2), we have

$$
\left\|\epsilon^{\mathfrak{n}}\right\|_{\infty} \leqslant\left\|I+D+\frac{1}{2} D^{2}\right\|_{\infty}\left\|\epsilon^{n-1}\right\|_{\infty}+\frac{\tau}{2}\|D\|_{\infty}\left(\beta_{1}\left\|\epsilon^{n-1}\right\|_{\infty}+\beta_{2}\left\|\epsilon^{n-m}\right\|_{\infty}\right)+\frac{\tau}{2}\left[\left(\beta_{1}\left\|\epsilon^{n-1}\right\|_{\infty}\right.\right.
$$




$$
\left.\left.+\beta_{2}\left\|\epsilon^{n-m}\right\|_{\infty}\right)+\beta_{1}\left(\|I+D\|_{\infty}\left\|\epsilon^{n-1}\right\|_{\infty}+\tau\left(\beta_{1}\left\|\epsilon^{n-1}\right\|_{\infty}+\beta_{2}\left\|\epsilon^{n-m}\right\|_{\infty}\right)\right)+\beta_{2}\left\|\epsilon^{n-m}\right\|_{\infty}\right] .
$$

Denoting $e^{n}=\max _{0 \leqslant i \leqslant n}\left\{\left\|\epsilon^{i}\right\|_{\infty}\right\}$, we have

$$
\begin{aligned}
e^{n} \leqslant & e^{n-1}+\frac{\tau}{2}\|D\|_{\infty}\left(\beta_{1} e^{n-1}+\beta_{2} e^{n-1}\right) \\
& +\frac{\tau}{2}\left[\left(\beta_{1} e^{n-1}+\beta_{2} e^{n-1}\right)+\beta_{1}\left(e^{n-1}+\tau\left(\beta_{1} e^{n-1}+\beta_{2} e^{n-1}\right)\right)+\beta_{2} e^{n-1}\right] \\
\leqslant & {\left[1+\tau\left(\beta_{1}+\beta_{2}\right)\left(1+\frac{1}{2}\left(\|D\|_{\infty}+\beta_{1} \tau\right)\right)\right] e^{n-1} \leqslant(1+\tau L) e^{n-1}, }
\end{aligned}
$$

where $L=\left(\beta_{1}+\beta_{2}\right)\left(1+\frac{1}{2}\left(\|D\|_{\infty}+\beta_{1} \tau\right)\right)$. Obviously, because of $\|I+D\|_{\infty} \leqslant 1,\|D\|_{\infty} \leqslant C_{0}<<+\infty$. Thus,

$$
e^{\mathrm{n}} \leqslant(1+\tau \mathrm{L})^{\mathrm{n}} e^{0} \leqslant e^{\mathrm{n} \tau \mathrm{L}} e^{0}=e^{\mathrm{TL}} e^{0} .
$$

Theorem 4.3 (Convergence). If $\|\mathrm{I}+\mathrm{D}\|_{\infty} \leqslant 1$, the method (3.3) is convergent.

Proof. Let $\mathrm{U}^{\mathrm{n}}$ and $\mathrm{u}^{\mathrm{n}}$ be the numerical and exact solution vectors, respectively, and

$$
u^{n}=\left[u\left(x_{1}, t_{n}\right), u\left(x_{2}, t_{n}\right), \ldots, u\left(x_{M-1}, t_{n}\right)\right]^{\top}, \quad \varepsilon^{n}=u_{i}^{n}-u\left(x_{i}, t_{n}\right) .
$$

Then,

$$
\begin{aligned}
\varepsilon^{n}= & \varepsilon^{n-1}+\frac{1}{2} D\left(2 \varepsilon^{n-1}+D \varepsilon^{n-1}+\tau\left(F\left(U^{n-1}\right)-F\left(u^{n-1}\right)\right)\right)+\frac{\tau}{2}\left[\left(F\left(u^{n-1}\right)\right.\right. \\
= & \left.\left.F\left(U^{n-1}+D u^{n-1}+\tau F\left(U^{n-1}\right)\right)\right)-\left(F\left(u^{n-1}\right)+F\left(u^{n-1}+D u^{n-1}+\tau F\left(u^{n-1}\right)\right)\right)\right]+O\left(\tau^{3}+\tau h^{2}\right) \\
= & \left(I+D+\frac{D^{2}}{2}\right) \varepsilon^{n-1}+\frac{\tau}{2} D\left(F\left(u^{n-1}\right)-F\left(u^{n-1}\right)\right)+\frac{\tau}{2}\left[F\left(u^{n-1}\right)-F\left(u^{n-1}\right)\right. \\
& \left.+F\left((I+D) U^{n-1}+\tau F\left(u^{n-1}\right)\right)-F\left((I+D) u^{n-1}+\tau F\left(u^{n-1}\right)\right)\right]+O\left(\tau^{3}+\tau h^{2}\right) .
\end{aligned}
$$

By means of the condition (1.2), we have

$$
\begin{aligned}
\left\|\varepsilon^{n}\right\|_{\infty} \leqslant & \left\|I+D+\frac{1}{2} D^{2}\right\|_{\infty}\left\|\varepsilon^{n-1}\right\|_{\infty}+\frac{\tau}{2}\|D\|_{\infty}\left(\beta_{1}\left\|\varepsilon^{n-1}\right\|_{\infty}+\beta_{2}\left\|\varepsilon^{n-m}\right\|_{\infty}\right)+\frac{\tau}{2}\left[\left(\beta_{1}\left\|\varepsilon^{n-1}\right\|_{\infty}+\beta_{2}\left\|\varepsilon^{n-m}\right\|_{\infty}\right)\right. \\
& \left.+\beta_{1}\left(\|I+D\|_{\infty}\left\|\varepsilon^{n-1}\right\|_{\infty}+\tau\left(\beta_{1}\left\|\varepsilon^{n-1}\right\|_{\infty}+\beta_{2}\left\|\varepsilon^{n-m}\right\|_{\infty}\right)\right)+\beta_{2}\left\|\varepsilon^{n-m}\right\|_{\infty}\right]+O\left(\tau^{3}+\tau h^{2}\right) .
\end{aligned}
$$

Denote $e^{n}=\max _{0 \leqslant i \leqslant n}\left\{\left\|\varepsilon^{i}\right\|_{\infty}\right\}$. Note that $\left\|I+D+\frac{1}{2} D^{2}\right\|_{\infty} \leqslant 1$, we have

$$
\begin{aligned}
e^{n} \leqslant & e^{n-1}+\frac{\tau}{2}\|D\|_{\infty}\left(\beta_{1} e^{n-1}+\beta_{2} e^{n-1}\right) \\
& +\frac{\tau}{2}\left[\left(\beta_{1} e^{n-1}+\beta_{2} e^{n-1}\right)+\beta_{1}\left(e^{n-1}+\tau\left(\beta_{1} e^{n-1}+\beta_{2} e^{n-1}\right)\right)+\beta_{2} e^{n-1}\right]+O\left(\tau^{3}+\tau h^{2}\right) \\
\leqslant & {\left[1+\tau\left(\beta_{1}+\beta_{2}\right)\left(1+\frac{1}{2}\left(\|D\|_{\infty}+\beta_{1} \tau\right)\right)\right] e^{n-1}+O\left(\tau^{3}+\tau h^{2}\right) \leqslant(1+\tau L) e^{n-1}+O\left(\tau^{3}+\tau h^{2}\right) }
\end{aligned}
$$

where $L=\left(\beta_{1}+\beta_{2}\right)\left(1+\frac{1}{2}\left(\|D\|_{\infty}+\beta_{1} \tau\right)\right)$. Thus,

$$
e^{\mathrm{n}} \leqslant(1+\tau \mathrm{L})^{\mathrm{n}} e^{0} \leqslant e^{\mathrm{n} \tau \mathrm{L}} e^{0}+\frac{\mathrm{O}\left(\tau^{3}+\tau \mathrm{h}^{2}\right)}{\tau \mathrm{L}}\left[(1+\tau \mathrm{L})^{\mathrm{n}}-1\right] .
$$

Note that $e^{0}=0$, then

$$
e^{n} \leqslant \frac{O\left(\tau^{3}+\tau h^{2}\right)}{\tau L}\left[(1+\tau L)^{n}-1\right] \leqslant \frac{O\left(\tau^{2}+h^{2}\right)}{L}\left[e^{T L}-1\right]=O\left(\tau^{2}+h^{2}\right)
$$

Remark 4.4. Let $\mu_{\alpha}=-\frac{\tau K_{\alpha} c_{\alpha}}{h^{\alpha}}$ and $B=A+A^{\top}$. Then, the matrix $I+D$ can be written as $I+D=I+\mu_{\alpha} B$. In the following, we discuss the range of value $\mu_{\alpha}$ when $\left\|I+\mu_{\alpha} B\right\| \leqslant 1$. 
If we take $M=40,200$ and investigate the range of value $\mu_{\alpha}$ when $\alpha$ varies from 1.1 to 2 , the numerical results can be shown in Fig. 1. From Fig. 1, we can see that the range of value $\mu_{\alpha}$ is dependent on $\alpha$, but it is almost independent of $M$. In this paper, we select $\mu_{\alpha}$ as shown in Table 1.
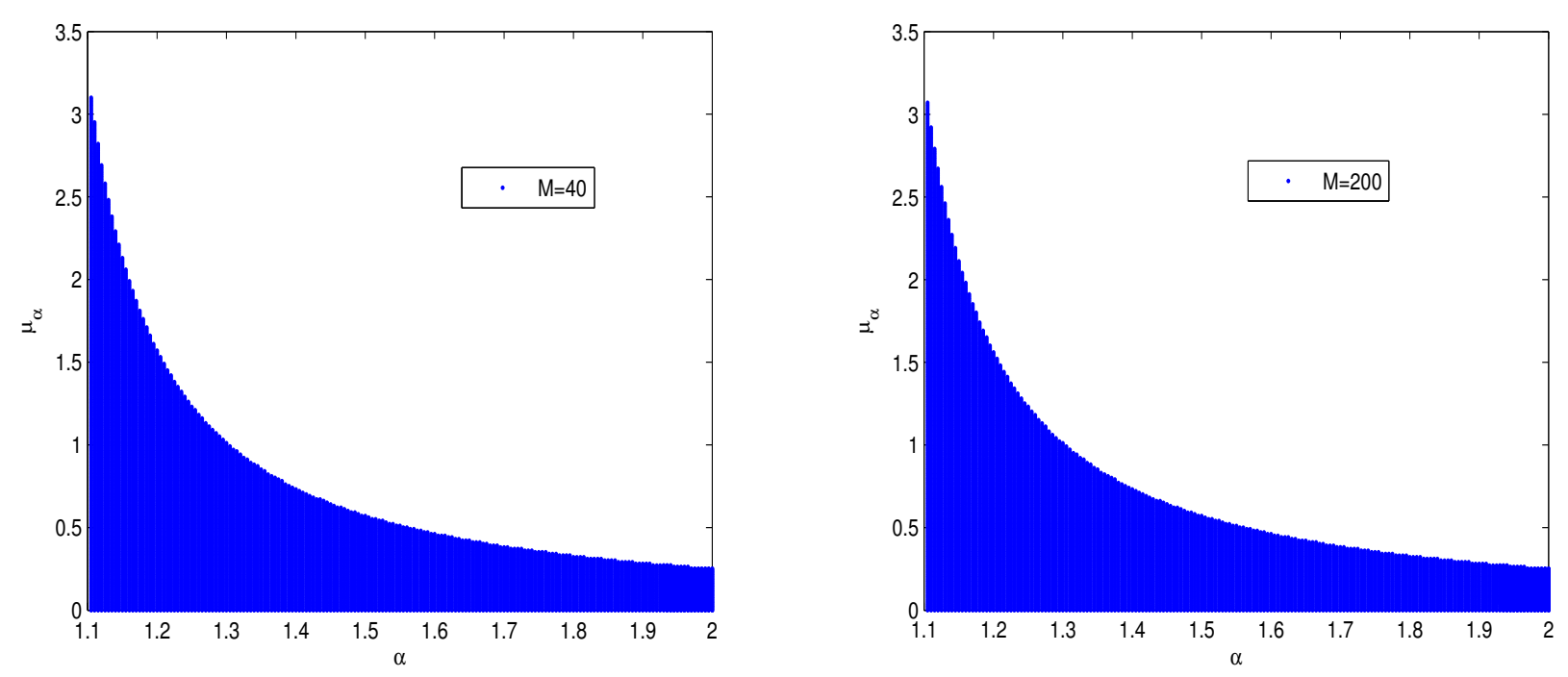

Figure 1: The range of value $\mu_{\alpha}$ when $\alpha$ varies from 1.1 to 2 .

\section{Numerical examples}

In order to demonstrate our theoretical results, we present some numerical examples in this section.

Example 5.1. Consider the following Riesz space fractional diffusion equations with delay:

$$
\left\{\begin{array}{l}
\frac{\partial u(x, t)}{\partial t}=K_{\alpha} \frac{\partial^{\alpha} u(x, t)}{\partial|x|^{\alpha}}+f(t, x, u(x, t), u(x, t-s)), \quad t \in[0, T] \\
u(x, t)=x^{2}(x-1)^{2} e^{-t}, t \in[-s, 0], 0 \leqslant x \leqslant 1 \\
u(0, t)=u(1, t)=0,0 \leqslant t \leqslant T
\end{array}\right.
$$

where $\mathrm{T}=10, \mathrm{~s}=1.0, \mathrm{~K}_{\alpha}=1,1<\alpha \leqslant 2$,

$$
\begin{aligned}
f(x, t, u(x, t), u(x, t-s))= & u(x, t) u(x, t-s)-x^{2}(1-x)^{2} e^{-t}-x^{4}(1-x)^{4} e^{-2 t+\tau} \\
& +\frac{e^{-t}}{2 \cos \left(\frac{\alpha \pi}{2}\right)}\left\{\frac{24}{\Gamma(5-\alpha)}\left[x^{4-\alpha}+(1-x)^{4-\alpha}\right]-\frac{12}{\Gamma(4-\alpha)}\left[x^{3-\alpha}+(1-x)^{3-\alpha}\right]\right. \\
& \left.+\frac{2}{\Gamma(3-\alpha)}\left[x^{2-\alpha}+(1-x)^{2-\alpha}\right]\right\},
\end{aligned}
$$

and the exact solution is $u(x, t)=x^{2}(1-x)^{2} e^{-t}$. 
For different $\alpha \in(1,2]$, the numerical solutions of (5.1) are obtained by using (3.3). When $\alpha=1.1$, the errors and the computing orders of (3.3) are shown in Table 2. When $\alpha=1.5$, we select $\mu_{\alpha}=0.57$, the corresponding numerical results are shown in Table 2. All of the numerical results show that the errors between the numerical solutions and the exact solutions are small and (3.3) for solving (1.1) is convergent and robust.

Table 2: The error and convergence order of the method (3.3).

\begin{tabular}{|c|c|c|c|c|c|c|c|}
\hline \multicolumn{4}{|c|}{$\alpha=1.1$} & \multicolumn{4}{|c|}{$\alpha=1.5$} \\
\hline$\tau$ & $\mathrm{h}$ & $\|e(h, \tau)\|_{\infty}$ & Order & $\tau$ & $\mathrm{h}$ & $\|e(h, \tau)\|_{\infty}$ & Order \\
\hline$\frac{1}{8}$ & $\frac{10}{200}$ & $1.5206 \mathrm{E}-03$ & & $\frac{1}{8}$ & $\frac{10}{280}$ & $2.2081 \mathrm{E}-03$ & \\
\hline$\frac{1}{16}$ & $\frac{10}{400}$ & $4.2502 \mathrm{E}-04$ & 1.81 & $\frac{1}{16}$ & $\frac{10}{793}$ & 4.5625E-04 & 2.27 \\
\hline$\frac{1}{32}$ & $\frac{10}{800}$ & 1.3587E-04 & 1.68 & $\frac{1}{32}$ & $\frac{10}{2245}$ & 1.1408E-04 & 2.00 \\
\hline$\frac{1}{64}$ & $\frac{10}{1600}$ & 4.2558E-05 & 1.67 & $\frac{1}{64}$ & $\frac{10}{6351}$ & 3.2659E-05 & 1.80 \\
\hline
\end{tabular}

Example 5.2. Consider the following Riesz space fractional diffusion equations with delay:

$$
\left\{\begin{array}{l}
\frac{\partial u(x, t)}{\partial t}=K_{\alpha} \frac{\partial^{\alpha} u(x, t)}{\partial|x|}+f(t, x, u(x, t), u(x, t-s)), \quad t \in[0, T], \\
u(x, t)=x^{2}(x-1)^{2} e^{-t}, \quad t \in[-s, 0], 0 \leqslant x \leqslant 1, \\
u(0, t)=u(1, t)=0,0 \leqslant t \leqslant T,
\end{array}\right.
$$

where $\mathrm{T}=10, \mathrm{~s}=0.5,1<\alpha \leqslant 2, \mathrm{~K}_{\alpha}=2$,

$$
\begin{aligned}
f(x, t, u(x, t), u(x, t-s))= & (u(x, t) u(x, t-s))^{2}-x^{2}(1-x)^{2} e^{-t}-x^{8}(1-x)^{8} e^{-4 t+1} \\
& +\frac{k_{\alpha} e^{-t}}{2 \cos \left(\frac{\alpha \pi}{2}\right)}\left\{\frac{24}{\Gamma(5-\alpha)}\left[x^{4-\alpha}+(1-x)^{4-\alpha}\right]-\frac{12}{\Gamma(4-\alpha)}\left[x^{3-\alpha}+(1-x)^{3-\alpha}\right]\right. \\
& \left.+\frac{2}{\Gamma(3-\alpha)}\left[x^{12-\alpha}+(1-x)^{2-\alpha}\right]\right\},
\end{aligned}
$$

and the exact solution is $u(x, t)=x^{2}(1-x)^{2} e^{-t}$.

For different $\alpha \in(1,2]$, the numerical solutions of (5.2) are obtained by using (3.3). When $\alpha=1.2$ and $\alpha=1.5$, we select $\mu_{\alpha}=1.56$ and $\mu_{\alpha}=0.57$, respectively, the errors and the computing orders of (3.3)

\begin{tabular}{|c|c|c|c|c|c|c|c|}
\hline \multicolumn{4}{|c|}{$\alpha=1.2$} & \multicolumn{4}{|c|}{$\alpha=1.5$} \\
\hline$\tau$ & $\mathrm{h}$ & $e(h, \tau) \|_{\infty}$ & Order & $\tau$ & $\mathrm{h}$ & $\mid e(h, \tau) \|_{\infty}$ & Order \\
\hline$\frac{1}{8}$ & $\frac{10}{251}$ & $2.0059 \mathrm{E}-03$ & & & $\frac{10}{561}$ & 2.7748E-03 & \\
\hline$\frac{1}{16}$ & $\frac{10}{577}$ & 4.9531E-04 & 2.02 & 16 & $\frac{10}{1587}$ & $6.2062 \mathrm{E}-04$ & 2.23 \\
\hline$\frac{1}{32}$ & $\frac{10}{1327}$ & $1.5728 \mathrm{E}-04$ & 1.66 & $\frac{1}{32}$ & $\frac{10}{4491}$ & $1.3245 \mathrm{E}-04$ & 2.23 \\
\hline$\frac{1}{64}$ & $\frac{10}{3050}$ & 4.2916E-05 & 1.87 & $\frac{1}{64}$ & $\frac{10}{12703}$ & 3.4513E-05 & 1.94 \\
\hline
\end{tabular}
are shown in Table 3. From the above computing results, we can find that the errors are also very small. Thus, we can draw the conclusion that (3.3) for solving (1.1) is stable and convergent.

Table 3: The error and convergence order of the method (3.3).

\section{Results and discussion}

In this research, the finite difference method which combines a time scheme based on the predictorcorrector method and the Crank-Nicolson scheme for the spatial discretization has been employed to solve a class of the Riesz space fractional diffusion equations with delay and a nonlinear source term. 
The corresponding theoretical results including stability and convergence are provided. Compared to the other implicit methods, the nonlinear system which is derived from discrete form of (1.1) does not have to be solved in this paper. The numerical results obtained by the proposed method confirm the robustness and the efficiency of it. And we hope that this work is a step in this direction.

\section{Acknowledgment}

This work is supported by projects from the National NSF of China (Grant No. 11501238, 11601180), NSF of Guangdong Province (Grant No.2016A030313119, 2014A030313641), and NSF of Huizhou University (Grant No. hzuxl201420).

\section{References}

[1] R. L. Bagley, P. J. Torvik, Fractional calculus in the transient analysis of viscoelastically damped structures, AIAA J., 23 (1985), 918-925. 1

[2] K. Diethelm, An algorithm for the numerical solution of differential equations of fractional order, Electron. Trans. Numer. Anal., 5 (1997), 1-6. 1

[3] K. Diethelm, N. J. Ford, Numerical solution of the Bagley-Torvik equation, BIT, 42 (2002), 490-507.

[4] K. Diethelm, N. J. Ford, A. D. Freed, A predictor-Corrector approach for the numerical solution of fractional differential equations, Nonlinear Dynam., 29 (2002), 3-22.

[5] K. Diethelm, G. Walz, Numerical solution of fractional order differential equations by extrapolation, Numer. Algorithms, 16 (1997), 231-253. 1

[6] M. Ichise, Y. Nagayanagi, T. Kojima, An analog simulation of non-integer order transfer functions for analysis of electrode processes, J. Electroanal. Chem., 33 (1971), 253-265. 1

[7] D. Kumar, R. P. Agarwal, J. Singh, A modified numerical scheme and convergence analysis for fractional model of Lienard's equation, J. Comput. Appl. Math., (2017), in press. 1

[8] D. Kumar, J. Singh, D. Baleanu, A fractional model of convective radial fins with temperature-dependent thermal conductivity, Romanian Reports in Physics, 2017 (2017), 13 Pages. 1

[9] D Kumar, J. Singh, D. Baleanu, Modified Kawahara equation within a fractional derivative with non-singular kernel, Therm. Sci., 2017 (2017), 10 Pages. 1

[10] F. Liu, V. Anh, I. Turner, Numerical solution of the space fractional Fokker-Planck equation, J. Comput. Appl. Math., 166 (2004), 209-219. 1

[11] F. Liu, P. Zhuang, V. Anh, I. Turner, K. Burrage, Stability and convergence of the difference methods for the space-time fractional advection-diffusion equation, Appl. Math. Comput., 191 (2007), 12-20.

[12] F. Liu, P. Zhuang, Q.-X. Liu, Numerical methods of fractional partial differential equations and their applications, Science Press (In Chinease), (2015). 1, 2.2, 2.3, 2.4, 4.1

[13] C. Lubich, Fractional linear multistep methods for Abel-Volterra integral equations of the second kind, Math. Comput., 45 (1985), 463-469.

[14] C. Lubich, Discretized fractional calculus, SIAM J. Math. Anal., 17 (1986), 704-719. 1

[15] R. L. Magin, Fractional calculus in bioengineering, Critical Reviews in Biomedical Engineering, 32 (2004), 1-104. 1

[16] M. M. Meerschaert, C. Tadjeran, Finite difference approximations for fractional advection-dispersion equations, J. Comput. Appl. Math., 172 (2004), 65-77. 1

[17] I. Podlubny, Fractional Differential Equations, Academic Press, New York, (1999). 1, 2.1

[18] J. Singh, D. Kumar, M. A. Qurashi, D. Baleanu, A new fractional model for giving up smoking dynamics, Adv. Difference Equ., 2017 (2017), 16 Pages. 1

[19] J. Singh, D. Kumar, M. A. Qurashi, D. Baleanu, A Novel Numerical Approach for a Nonlinear Fractional Dynamical Model of Interpersonal and Romantic Relationships, Entropy, 2017 (2017), 17 Pages. 1

[20] H. H. Sun, A. Abdelwahab, B. Onaral, Linear approximation of transfer function with a pole of fractional order, IEEE Trans. Automat. Contr., 29 (1984), 441-444. 1

[21] H. H. Sun, B. Onaral, Y. Tsao, Application of positive reality principle to metal electrode linear polarization phenomena, IEEE. Trans. Biomed. Eng., 31 (1984), 664-674.

[22] V. V. Uchaikin, R. T. Sibatov, Fractional theory for transport in disordered semiconductors, Commun. Nonlinear Sci. Numer. Simul., 13 (2008), 715-727. 1

[23] G.-C. Wu, D. Baleanu, Z.-G. Deng, S.-D. Zeng, Lattice fractional diffusion equation in terms of a Riesz-Caputo difference, Phys. A, 438 (2015), 335-339. 1

[24] G.-C. Wu, D. Baleanu, W.-H. Luo, Lyapunov functions for Riemann-Liouville-like fractional difference equations, Appl. Math. Comput., 314 (2017), 228-236.

[25] G.-C. Wu, D. Baleanu, H.-P. Xie, Riesz Riemann-Liouville difference on discrete domains, Chaos, 26 (2016), 5 Pages. 1 
[26] X. Zhao, Z.-Z. Sun, G. E. Karniadakis, Second-order approximations for variable order fractional derivatives: Algorithms and applications, J. Comput. Phys., 293 (2015), 184-200. 1

[27] P. Zhuang, F. Liu, V. Anh, I. Turner, Numerical methods for the variable-order fractional advection-diffusion equation with a nonlinear source term, SIAM J. Numer. Anal., 47 (2009), 1760-1781. 1 